\title{
L'attractivité des territoires dans un contexte de mondialisation : quel espoir pour les milieux ruraux de l'Outaouais?
}

\author{
Guy Chiasson \\ Université du Québec en Outaouais
}

Le concept d'attractivité, lorsqu'il est utilisé par les chercheurs et intervenants préoccupés par le développement des territoires, renvoie autant à la capacité de ces territoires d'attirer des personnes et de l'activité économique qu'à celle de les retenir. Cette attractivité est, depuis très longtemps, un défi de taille pour les milieux ruraux québécois. On n'a qu'à penser à la problématique séculaire de «l'exode rural» pour se rendre compte que la difficulté de retenir les populations, au moins celles jugées les plus dynamiques, ne date pas d'hier. Cependant, on peut assez facilement convenir que le présent contexte de mondialisation introduit de nouvelles dynamiques qui bouleversent le potentiel attractif des territoires, tout spécialement celui des milieux ruraux.

La mondialisation, on le répète souvent, est marquée par une économie où la concurrence est de plus en plus sévère parce ce qu'elle ne bénéficie plus de la protection des États nationaux. Dans ce contexte fortement compétitif, la «nouvelle économie », c'est-àdire une économie où la composante innovation et savoir-faire est importante, devient une clé incontournable du succès des entreprises, mais aussi des territoires ${ }^{1}$. L'attractivité des localités et des régions dépend assez largement, bien que pas exclusivement ${ }^{2}$, de leur capacité à relever ce défi de la nouvelle économie du savoir.

Selon une hypothèse très courante, le passage à la dynamique de nouvelle économie instaurerait une hiérarchie spatiale très claire. Au sommet de la hiérarchie, on retrouve les villes métropoles, et plus particulièrement celles qui ont une portée internationale. Celles-ci seraient les mieux équipées pour soutenir les innovations économiques qui sont le gage de succès dans la nouvelle économie. La concentration des universités et des centres de recherche, la présence d'une main-d'œuvre diversifiée et qualifiée, l'accès à de l'infrastructure technologique de pointe, etc. sont des facteurs qui se conjuguent pour faire de ces métropoles des «pépinières de la nouvelle économie " ${ }^{3}$. $\mathrm{Au}$ bas de la hiérarchie se trouveraient les milieux ruraux et les villes plus modestes qui sont en quelque sorte condamnés à se partager des activités économiques nettement moins intéressantes parce que très limitées du point de vue de la valeur ajoutée. Ce processus de hiérarchisation, que d'aucuns qualifient de "métropolisation », mènerait à la marginalisation économique des milieux ruraux et à leur exclusion des réseaux et circuits d'échange dominants. Ces tendances - celles instaurées par la mondialisation - ne manquent pas d'avoir des conséquences graves sur l'attractivité des milieux ruraux : exode des jeunes mais aussi parfois des retraités en quête d'emplois et de services, fluctuation de l'activité économique locale en fonction des soubresauts des marchés mondiaux des matières premières, fragilité des services locaux, etc. Ces conséquences sont trop bien connues des ruraux.

Ce texte veut se pencher sur l'attractivité dans le cas des milieux ruraux de l'Outaouais. Pour ce faire, je prendrai comme point de départ l'hypothèse de la métropolisation que je viens d'expliciter. Est-ce que cette dynamique de métropolisation colle à la réalité de l'Outaouais rural ? Est-ce que les territoires ruraux de l'Outaouais sont condamnés à s'accrocher à des activités économiques déclinantes et donc peu porteuses en matière d'attractivité ? 
Afin d'apporter des éléments de réponse à cette question, mon analyse se fera sur deux plans. Tout d'abord, je vais tracer un portrait global de la situation économique des trois MRC rurales périphériques (Vallée de la Gatineau, Papineau et Pontiac). La comparaison avec l'Outaouais urbain (Ville de Gatineau) et périurbain (la MRC des Collines de l'Outaouais) permettra de montrer que, d'un point de vue d'ensemble, la thèse de la métropolisation semble se confirmer: les milieux ruraux outaouais restent fortement rattachés à des productions déclassées et peu porteuses de valeur ajoutée. Cette lecture assez pessimiste de la situation me semble tout de même devoir être nuancée, ce que je vais faire dans un deuxième temps. $\mathrm{Si}$, à l'échelle globale, les disparités d'attractivité entre milieux ruraux et milieux plus centraux sont frappants, on peut tout de même identifier à des échelles plus localisées des expériences qui sont prometteuses parce qu'elles initient la possibilité d'une nouvelle économie rurale et d'un «développement rural durable ». Je vais plus précisément me pencher sur une voie qui m'apparaît prometteuse dans le cas de l'Outaouais, la réappropriation du potentiel forestier par les communautés locales.

\section{Le cas de l'Outaouais rural québécois}

La région de l'Outaouais, prise dans son ensemble, s'en tire assez avantageusement en matière d'attractivité. Ce constat vaut autant pour la capacité d'attirer des personnes que de l'activité économique. Selon des données de l'Institut québécois de la statistique colligées par des collègues de l'Université du Québec en Outaouais ${ }^{4}$, la région affiche pour la période de 1986 à 1996 des taux de croissance démographique de plus de $18 \%$, comparativement à la moyenne québécoise qui est d'environ $9 \%$. Les taux de chômage de la région pour 2001 sont également en deçà de la moyenne québécoise, tandis que les taux d'activité (67\%) y sont significativement plus élevés $(63,6 \%$ pour l'ensemble du Québec).

Cette activité économique soutenue s'explique en bonne partie par la force du secteur tertiaire de la région. Depuis les années 1970 et les politiques trudeauistes de bilinguisation de la fonction publique, le gouvernement fédéral a relocalisé une partie importante de ses activités sur le territoire urbanisé de l'Outaouais. Cette relocalisation a coïncidé à peu près avec l'effondrement de l'industrie papetière comme moteur industriel, de sorte que le secteur public est devenu l'épine dorsale de l'économie régionale. De surcroît, au début des années 1990, face à un ralentissement de l'emploi dans la fonction publique fédérale, des efforts assez importants ont été consentis pour diversifier l'économie de la région vers le secteur des nouvelles technologies de l'information et de la communication. Ces efforts ${ }^{5}$ ne sont venus que confirmer la très grande place du secteur des services, de sorte qu'avec $84,5 \%$ de sa main-d'œuvre employée dans le secteur tertiaire, incluant plus de 33000 travailleurs qui œuvrent dans l'administration publique, l'Outaouais est la région la plus tertiarisée du Québec (la moyenne québécoise est de moins de $75 \%$ ). Cette tendance nette à la tertiarisation de l'économie régionale ne semble pas sur le point de fléchir puisque, entre 1991 et 1996, les activités primaires et secondaires ont reculé significativement dans l'emploi total de la région au profit de la croissance des activités de service.

\section{Cette activité économique soutenue s'explique en bonne partie par la force du secteur tertiaire de la région.}

Ces quelques indicateurs laissent bien voir la capacité $\mathrm{du}$ secteur tertiaire à jouer un rôle moteur pour l'attractivité du territoire régional. C'est la croissance forte des activités de services publics et privés, plutôt que les secteurs primaires et secondaires déclinants, qui explique les performances soutenues de l'économie outaouaise. C'est sans doute également cette force du tertiaire qui explique les soldes migratoires positifs qu'à su afficher la région depuis plusieurs décennies ainsi que la capacité d'attirer des travailleurs qualifiés provenant de nombreuses autres régions du Québec.

\section{Des décalages importants entre le rural et le métropolitain}

Ces constats très positifs en matière d'attractivité caractérisent bien la portion plus métropolitaine de l'Outaouais, c'est-à-dire le territoire de la nouvelle Ville de Gatineau ${ }^{6}$ ainsi que celui de la MRC des Collines de l'Outaouais (un territoire assez largement périurbain qui bénéficie à plusieurs égards de sa proximité de Gatineau) ${ }^{7}$. Ces chiffres cachent cependant de fortes disparités entre les portions rurale et 
métropolitaine du territoire. Ils ne laissent pas voir les difficultés propres aux milieux ruraux dans un contexte de passage à la nouvelle économie tertiarisée. Ces difficultés transparaissent peu dans les statistiques globales étant donné justement la faiblesse démographique du rural par rapport à l'ensemble régional.

Dans l'espace rural (les MRC de la Vallée de la Gatineau, du Pontiac et de Papineau) plus périphérique par rapport au cœur métropolitain, les indicateurs de l'attractivité sont beaucoup moins favorables. Quelques exemples empruntés encore une fois au portrait de l'économie et de la démographie régionale suffi- ront pour montrer les décalages importants entre la réalité urbaine et rurale. Pour la période de 1996, les taux d'activités pour ces trois MRC (entre $52 \%$ et $57 \%$ ) sont significativement en deçà de la moyenne provinciale $(62 \%)$. La Ville de Gatineau ainsi que la MRC des Collines de l'Outaouais affichent respectivement des taux d'activités de $69 \%$ et $70 \%$. Du point de vue démographique, les trois MRC rurales de l'Outaouais font face à une problématique de migration des jeunes qui contribue, comme le montre le tableau 1 , à un vieillissement nettement plus prononcé de la population des ces sous-régions comparativement à la Ville de Gatineau.

\section{Tableau 1 - Âge moyen des résidents de l'Outaouais par MRC}

\begin{tabular}{|c|c|c|c|c|}
\hline Ville de Gatineau & $\begin{array}{c}\text { Collines de } \\
\text { l'Outaouais }\end{array}$ & $\begin{array}{c}\text { Vallée de la } \\
\text { Gatineau }\end{array}$ & Papineau & Pontiac \\
\hline 34 ans & 33,7 ans & 38.2 ans & 39,6 ans & 38,1 ans \\
\hline
\end{tabular}

Comme on peut s'y attendre, l'emploi tertiaire est, dans les MRC périphériques, moins présent que dans la portion urbanisé. On n'y retrouve pas cette forte présence du secteur public et encore moins cette incursion dans le secteur des technologies de l'information et des communications (TIC). Les territoires ruraux ont gardé une dépendance accrue envers les activités traditionnelles d'extraction et de première transformation des matières premières. Des activités comme la récolte de la matière ligneuse forestière, le sciage, l'élevage du bœuf de boucherie continuent de marquer l'économie de ces territoires de façon très significative.

\section{Une fuite de valeur ajoutée}

La place encore importante qui est réservée aux secteurs traditionnels forestiers et agricoles n'est pas en soi problématique. Elle le devient dans la mesure où une grande partie de la richesse produite par ces activités est en quelque sorte exportée hors de la région. Que ce soit au plan de l'exploitation forestière ou de celui de l'agriculture, la production rurale outaouaise a tendance à s'arrêter à la première transformation et mise en valeur de la ressource. L'Outaouais est bien pourvu au plan des usines de sciage qui sont, pour la plupart, situées hors du territoire métropolitain, mais

compte très peu d'activités au niveau de la deuxième et troisième transformation du bois qui sont plus productrices de richesses.

Même constat dans le cas de l'agriculture. L'élevage, qui prend en Outaouais une place très importante dans l'agriculture de la région, s'est concentré dans la production de veaux qui sont en bonne partie exportés pour la finition. Dans un cas comme dans l'autre, l'économie des territoires de l'Outaouais laisse s'échapper, en quelque sorte, une partie significative des productions plus spécialisées et de la valeur ajoutée.

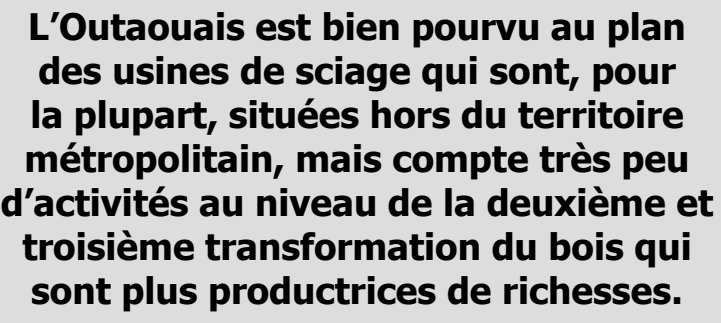

Il faut dire que l'économie tertiaire est loin d'être absente dans ces milieux ruraux. En fait, dans les trois MRC dont il est question ici, la présence de ce secteur est quand même prédominante puisqu'elle dépasse les $50 \%$. Cela est attribuable, d'une part, à une activité 
de villégiature importante (chasse et pêche, chalets, etc.) et surtout, d'autre part, à la présence de services administratifs publics concentrés dans les chefs-lieux des municipalités (hôpitaux, bureaux locaux de certains ministères, etc.) ou plus déconcentrés sur le territoire (écoles primaires, services municipaux). Ces activités ont assurément servi de frein à la dévitalisation mais n'ont pu se suppléer à des activités privées déclinantes et servir de moteur à une attractivité forte pour le territoire dans son ensemble.

\section{Une voie prometteuse : la maîtrise locale des forêts}

L'analyse que j'ai pu faire jusqu'à présent met à découvert les mécanismes de métropolisation tels qu'ils prennent place dans le cas de l'Outaouais rural : concentration des activités de pointe dans les territoires centraux capable de mobiliser les ressources importantes pour l'économie du savoir et "spécialisation » de la périphérie rurale dans des activités plus banales (qu'elles soient primaire, secondaire ou même tertiaire) beaucoup moins porteuses de valeur ajoutée et d'attractivité. Ce portrait assez pessimiste face à l'attractivité des milieux ruraux de l'Outaouais que j'ai esquissé à grands traits ne laisse-t-il aucun espoir pour les milieux ruraux?

\section{L'Outaouais rural compte quelques expériences qui, si elles n'ont pas réussi à infléchir globalement la tendance historique au déclin du rural, sont tout de même prometteuses du point de vue de l'attractivité des micro-territoires.}

Ce type d'analyse qui insiste sur la marginalisation des milieux ruraux périphériques est d'ailleurs très courant, que ce soit au Québec ${ }^{8}$ ou ailleurs. Je considère cependant qu'il doit être nuancé. L'Outaouais rural compte quelques expériences qui, si elles n'ont pas réussi à infléchir globalement la tendance historique au déclin du rural, sont tout de même prometteuses du point de vue de l'attractivité des microterritoires. À l'instar de plusieurs spécialistes de la ruralité $^{9}$, je considère que ces voies prometteuses méritent d'être analysées ne serait-ce qu'afin de tempérer les constats pessimistes sur la ruralité dans un contexte de mondialisation. Je terminerai donc ce tex- te par la présentation d'une de ces voies, la réappropriation du patrimoine forestier par les communautés locales.

La forêt outaouaise, même si elle a été comme ailleurs écrémée ${ }^{10}$, représente un potentiel de développement intéressant, notamment du fait de sa grande diversité (présence d'une multiplicité d'essences de feuillus). À cause d'un modèle d'exploitation peu diversifié centré sur la première transformation des bois (le sciage) pour l'exportation et dominé (dans le cas de la forêt publique) par les compagnies forestières et l'État, la maîtrise de ce potentiel échappe encore largement aux communautés locales. Résultat, même si elles sont situées à proximité d'une richesse forestière assez abondante, les communautés locales restent dans un état de dépendance face à la forêt. C'est-à-dire qu'elle en bénéficie assez peu en termes de développement et qu'elles ont peu à dire sur les façons de l'aménager et de la mettre en valeur.

Face à un modèle d'exploitation qui est entré en crise, plusieurs réclament une meilleure participation des communautés rurales à la gestion et aux bénéfices tirés de la forêt. C'est dans ce contexte qu'ont a été instituées la Forêt de l'Aigle en 1996 et la Corporation qui est appelée à en assurer la gestion et la mise en valeur. Le mode de gestion particulier de ce territoire forestier de 141 hectares situé à cheval sur la MRC de la Vallée de la Gatineau et celle du Pontiac va nettement dans le sens d'une recherche de nouveaux rapports entre les forêts publiques et les communautés locales.

Cette expérience, qui s'est inspirée du concept de « forêt habitée », est reconnue par plusieurs comme un modèle pour un renouvellement innovateur du modèle dominant d'exploitation forestière ${ }^{11}$. La Corporation de gestion de la Forêt de l'Aigle a en effet institué un mode de gestion partenarial et décentralisé du patrimoine forestier faisant appel à divers usagers (chasse et pêche, pourvoiries, Autochtones, industriels, environnementalistes, monde municipal, etc.). En incorporant de la sorte différents parties prenantes, l'expérience cherche à stimuler le développement d'une compréhension élargie du potentiel forestier qui est loin de se limiter à la simple matière ligneuse reconnue par les modes d'exploitation traditionnels.

Le caractère innovateur de cette initiative est double. D'une part, elle a permis de développer de nouvelles 
expertises et pratiques en ce qui a trait à la gestion et à la mise en valeur de la filière matière ligneuse. La gestion concertée a permis le renouvellement des pratiques en insistant sur l'aménagement et la formation de la main-d'œuvre. En plus d'instituer par l'aménagement des formes durables de récoltes de la matière ligneuse et l'approvisionnement aux usines de sciage locales, l'initiative de la Forêt de l'Aigle permet aussi d'utiliser la ressource forestière comme un levier pour développer des formes de travail plus qualifiées.

D'autre part, la Corporation a initié un processus visant à répertorier le potentiel d'un point de vue multidimensionnel (flore, faunique en plus du potentiel des arbres) de la Forêt de l'Aigle. Cet exercice ouvre ainsi la porte à l'identification de nouvelles utilisations productives de la forêt qui avaient été auparavant négligées (plantes médecinales, par exemple) même si elles sont porteuses de richesses.

Le cas de la Corporation de la Forêt de l'Aigle est prometteur dans le sens où il permet de revaloriser le potentiel forestier et de le requalifier en faisant appel à des activités plus spécialisés de travail forestier. Il est utile de rappeler que ce rapport différent avec la forêt a été rendu possible par la participation partenariales des divers intervenants provenant de ces communautés. Ce décloisonnement de la gestion forestière, qui fait que les compagnies forestières sont un intervenant parmi plusieurs intervenants provenant des communautés, semble donc une condition nécessaire pour assurer une telle requalification de la forêt.

Si l'expérience de la Forêt de l'Aigle est encore jeune, on peut penser qu'elle aura des conséquences favorables sur la contribution de la forêt à l'attractivité des communautés situées à sa proximité. La réappropriation des forêts comme celle initiée par la Corporation de la Forêt de l'Aigle est intéressante pour l'Outaouais rural, mais aussi de façon générale pour les régions rurales périphériques québécoises. Cet intérêt vient justement du fait qu'en prenant place dans le secteur forestier, une telle initiative peut s'appuyer en bonne partie sur des ressources déjà en place dans les territoires ruraux. En effet, ces milieux ont non seulement accès à des ressources naturelles, mais à cause d'une longue expérience avec la forêt, elles ont également de l'infrastructure, de la main-d'œuvre et une certaine expertise sur lesquelles elles peuvent tabler pour se positionner dans la nouvelle économie. Délaisser totalement le secteur des ressources naturel- les pour miser sur des activités totalement nouvelles dans des secteurs de pointe (NTIC, par exemple) aurait comme désavantage net de déqualifier d'avance une partie de la population ou de la condamner à œuvrer dans des secteurs en perte de vitesse.

\section{En prenant place dans le secteur forestier, une telle initiative peut s'appuyer en bonne partie sur des ressources déjà en place dans les territoires ruraux. En effet, ces milieux ont non seulement accès à des ressources naturelles, mais à cause d'une longue expérience avec la forêt, elles ont également de l'infrastructure, de la main-d'œuvre et une certaine expertise sur lesquelles elles peuvent tabler pour se positionner dans la nouvelle économie.}

\section{Notes et références}

1 Bon nombre de travaux contemporains consacrés à l'innovation productive dans un contexte de nouvelle économie considèrent que celle-ci n'est pas le produit exclusif des entreprises, mais qu'elle est aussi en bonne partie redevable des qualités particulières des milieux géographiques et de la richesse des relations que peuvent entretenir les entrepreneurs avec leur milieux. On peut citer à titre d'exemples, parmi les travaux sensibles à la portée innovatrice des territoires, ceux qui font appel au concept « milieux innovateurs ». Pour une recension des écrits récentes sur la question de l'innovation dans les communautés, et tout particulièrement les communautés rurales, voir la recension récente de Pierre-Marcel Desjardins (2003). Une communauté rurale innovante et performante: quels sont les facteurs pertinents? Moncton, Institut canadien de recherche sur le développement régional, $27 \mathrm{p}$.

2 Comme le fait valoir le courant français de l' « économie résidentielle », plusieurs facteurs incluant les politiques redistributives de l'État (transferts sociaux, etc.) continuent de stabiliser la démographie de milieux qui devraient pourtant se désertifier, si l'on se fie uniquement au dynamisme de leurs activités productives.

3 J'emprunte ici la belle expression de Pierre Veltz (1999). «Métropoles, périphéries et économie mondiale », dans Jean-Marc Fontan, Juan-Luis Klein et Diane-Gabrielle Tremblay (dir.), Entre la métropolisation et le village global, Québec, Presses de l'Université du Québec, 325 p.

4 Je m'appuie ici sur deux documents préparés par la Chaire de recherche en développement des collectivités (CRDC) de l'Université du Québec en Outaouais qui cherchent à 
faire le portrait de l'Outaouais. Le premier fait le portrait des tendances démographiques de cette région tandis que le second dépeint les tendances économiques récentes de cette région : 1) Doucet, Chantale (2002). Portrait démographique de l'Outaouais et de ses sous-régions, sous la direction de Louis Favreau et Martin Robitaille, Cahier de la Chaire de recherche en développement des collectivités, $\mathrm{n}^{\circ} 2,49$ p.; 2) Doucet, Chantal, (à paraître). Portrait économique de l'Outaouais, sous la direction de Louis Favreau et Martin Robitaille, Cahier de la Chaire de recherche en développement communautaire.

5 Parmi ces efforts, on peut penser notamment à ceux déployés par la Société de diversification économique que l'Outaouais (SDEO) a créée spécifiquement pour trouver des alternatives à l'emploi dans la fonction publique.

6 On se souviendra qu'à la suite des récentes fusions municipales, la nouvelle ville de Gatineau a été constituée à partir des anciennes villes d'Aylmer, Hull, Gatineau, Masson-Angers et Buckingham.

7 La Ville de Gatineau est composée d'environ 225000 habitants, ce qui n'en fait pas nécessairement en soi un espace métropolitain de grande envergure. Cependant, elle s'inscrit, quoique de façon quelque peu conflictuelle, dans un dynamique métropolitaine plus large, celle de l'agglomération d'Ottawa-Gatineau, la quatrième en importance en termes de population au Canada.

8 Pour ne citer que cet exemple, Clermont Dugas a montré pour l'ensemble du Québec les tendances que j'ai pu dégager ici pour l'Outaouais : l'enfoncement et la marginalisation du rural profond et la montée en force des milieux ru- raux proches des grandes villes. Voir notamment Clermont Dugas (2000). «L'espace rural québécois », dans Mario Carrier et Serge Côté (dir.), Gouvernance et territoires ruraux. Éléments d'un débat sur la responsabilité du développement, Québec, Presses de l'Université du Québec, p. $13-40$.

9 Je pense ici notamment aux travaux de Bruno Jean qui ont montré avec force qu'un développement rural « durable » et « approprié » est possible et que des expériences dynamiques et innovatrices allant dans ce sens sont déjà en place. Je partage également avec cet auteur le constat voulant que la présence d'expériences prometteuses du point de vue d'un renforcement de l'attractivité rurale ne signifie aucunement que celles-ci peuvent et doivent se passer de politiques publiques spéciales soutenant ces efforts. Voir Bruno Jean (1996). Territoires d'avenir, Québec, Presses de l'Université du Québec; Bruno Jean (2002). « Réussir le développement approprié des régions rurales. Quelques conditions gagnantes "), dans Louis Favreau, Martin Robitaille et Daniel Tremblay (dir.), Quel avenir pour les régions?, Gatineau, Chaire de recherche en développement des collectivités, p. 81-94.

10 Les grandes forêts de pins blancs géants qui marquaient le territoire de l'Outaouais il y a quelques siècles ont été décimées de sorte que cette essence est à peu près disparue aujourd'hui.

11 Sur la Forêt de l'Aigle, voir le commentaire de Charles de Blois Martin qui qualifie cette expérience de «laboratoire d'une foresterie nouvelle». Charles de Blois Martin (2002). "Émergence d'une nouvelle économie rurale », dans Annuaire du Québec 2003, Montréal, Fides, p. 241259. 